\title{
VIRGILIO, OVIDIO Y, QUIZÁ, VALERIO FLACO EN UN NUEVO FRAGMENTO DE INSCRIPCIÓN ROMANA PROCEDENTE DE TARRACO
}

\author{
DIANA GOROSTIDI PI* \\ Universitat Rovira $i$ Virgili - Institut Català d'Arqueologia Clàssica \\ dgorostidi@icac.cat \\ ORCID: 0000-0002-5550-7920
}

\section{RESUMEN}

Presentamos un nuevo fragmento de inscripción funeraria procedente de la antigua Tarraco con parte de un carmen epigraphicum en hexámetros dactílicos. A pesar del poco texto conservado, el contenido permite identificar un centón a partir del repertorio poético de Virgilio y Ovidio, y quizá también de Valerio Flaco.

PALABRAS CLAVE: centón, Ovidio y Virgilio, Valerio Flaco, carmen epigraphicum, Tarraco, poesía funeraria.

\section{VIRGIL, OVID, AND MAYBE VALERIUS FLACCUS IN A NEW FRAGMENTARY ROMAN INSCRIPTION FROM TARRACO}

\section{ABSTRACT}

A new fragmentary funerary inscription from Tarraco containing part of a carmen epigraphicum is presented. Although the preserved text is short, the contents allow to identify a cento from Virgil's and Ovid's poetic repertory, and maybe also from Valerius Flaccus'.

KEYWORDS: cento, Ovid and Virgil, Valerius Flaccus, carmen epigraphicum, Tarraco, funerary poetry.

Presentamos en esta sede una nueva inscripción romana fragmentaria procedente de Tarragona con parte de un carmen epigraphicum. Fue hallada por casualidad en un lugar indefinido de la parte alta de la ciudad. ${ }^{1}$ Del carácter funerario de su contenido es posible proponer un emplazamiento original en el área sepulcral del llamado suburbio oriental de la antigua Tarraco, situado en torno al paso de la vía Augusta procedente de Barcino (cf. Ciurana 2011: 334-336).

\section{LA INSCRIPCIÓN}

Se trata de un bloque de la característica caliza local, llamada "piedra de Santa Tecla", en su variedad rosada. Actualmente está roto por sus cuatro costados (27)

\footnotetext{
* Este trabajo se enmarca en el proyecto Officinae (HAR2015-65379-P, MINECO/FEDER,UE).

${ }^{1}$ No hay más detalles sobre su localización. La inscripción llegó a través de un particular a manos de los gestores de la empresa «A Priori Cultural», de Tarragona, quienes se dispusieron a depositarla en el Museo Nacional Arqueológico de Tarragona. Agradezco a Cristina Benet, gerente de la empresa, su amable invitación a estudiar esta pieza. La autopsia se realizó el día 17 de julio del 2019 en el laboratorio de la empresa.
} 
x (31) x $14 \mathrm{~cm}$ (fig.1). En su cara frontal se encuentra una inscripción latina de siete líneas, las tres primeras con letras capital cuadrada de módulo notablemente mayor. Un espacio de $4 \mathrm{~cm}$ separa visualmente esta primera parte de la segunda, donde se halla el carmen epigraphicum, cuyas cuatro líneas conservadas están repartidas en una secuencia formada por un primer verso separado de un grupo de dos, seguido a su vez por un cuarto verso, que desconocemos si iba solo o emparejado con un quinto, dado que por desgracia la inscripción se trunca en este punto.

Las letras están incisas también en capital cuadrada, con tendencia a la actuaria, de módulo mucho más pequeño y mejor ejecutado, probablemente por una segunda mano ${ }^{2}$. De la última línea solo se conserva la parte superior de algunas letras. La rotura de lateral derecho no ha afectado el final de las líneas, pero la erosión de la superficie afecta a la lectura de la mitad de las líneas 5 y 6 . Alt. Letras: líneas 1-3: $3 \mathrm{~cm}$ (O de 3,3 cm en la 1.3); líneas 4-6: $1 \mathrm{~cm}$ (I longa de 1,6 $\mathrm{cm}$ en la 1.4); C ligeramente divergente en 1.1; T montante en la 1.7. La última línea está partida por la parte superior y apenas se distinguen trazos de letras aislados. Las interpunciones tienen forma de vírgula y destaca la presencia de hederae distinguentes ornamentales de gran tamaño al final de las líneas 2 y 3 .

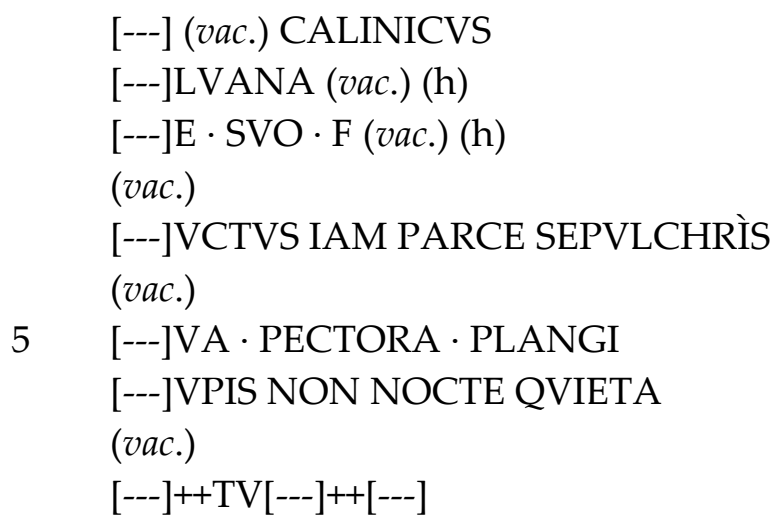

L.1. De lectura clara, a pesar de que las cuatro primeras letras conservadas estén partidas por la mitad.

L.4. Juctus: a simple vista puede ser leído como Jucius, pero la luz rasante ha confirmado el largo trazo horizontal de una $\mathrm{T}$.

L.6. Un golpe superficial sobre la segunda letra $(\mathrm{P})$ crea la ilusión óptica de un trazo oblicuo, lo que puede dar la impresión de que se trata de una R. Sin

2 Este uso de diversos tamaños en las letras, que combina incluso con tipos de caligrafía diferentes, como en este caso, es un recurso habitual y bien documentado en este tipo de inscripciones y servía, más allá de las funciones estéticas, para diferenciar el cuerpo de la inscripción funeraria en prosa de la parte con la composición métrica o simplemente poética (Limón 2014: 96-97). Existen paralelos en Tarraco con una distribución del texto en diversas franjas, mayor la parte en prosa con los nombres de los personajes: CLE 981 (= CIL II 4428) y CIL II²/14, 2301 (= IRAT $23=$ AE 2016, 894), ambas datadas en el s. I d.C. 
embargo, la lectura con la luz rasante ha confirmado la P. Por otro lado, el refuerzo horizontal del palo podría inducir a interpretar este carácter como un nexo LP ([---c]ulpis), pero el estudio del texto ha permitido descartar esta lectura a favor de cupis.

L.7: La primera crux corresponde a una letra redonda, probablemente una $\mathrm{O}$, y la segunda una $\mathrm{P}$ o una R. Después de la T montante se aprecian los extremos de los trazos oblicuos de una $\mathrm{V}$, por lo que la secuencia puede reconstruirse como ORTV. La siguiente crux corresponde a una $\mathrm{R}$ o una $\mathrm{B}$, mientras la última crux es el extremo de un astil vertical, probablemente una I.

Lectura:

------ / [---] Cal(l)inicus / [--- S]ilvana / [--- d]e suo f(ecerunt).

[--- l]uctus iam parce sepulchris

[--- s]ua pectora plangi

[--- cu]pis non nocte quieta

[--- ]ọtu[---]++[---]

1.7: Hay diversas opciones que podrían considerarse como propuestas de lectura, entre ellas ortus, fortuna, mortuus, portus, hortus y sus derivados, que son las más habituales en los CLE.

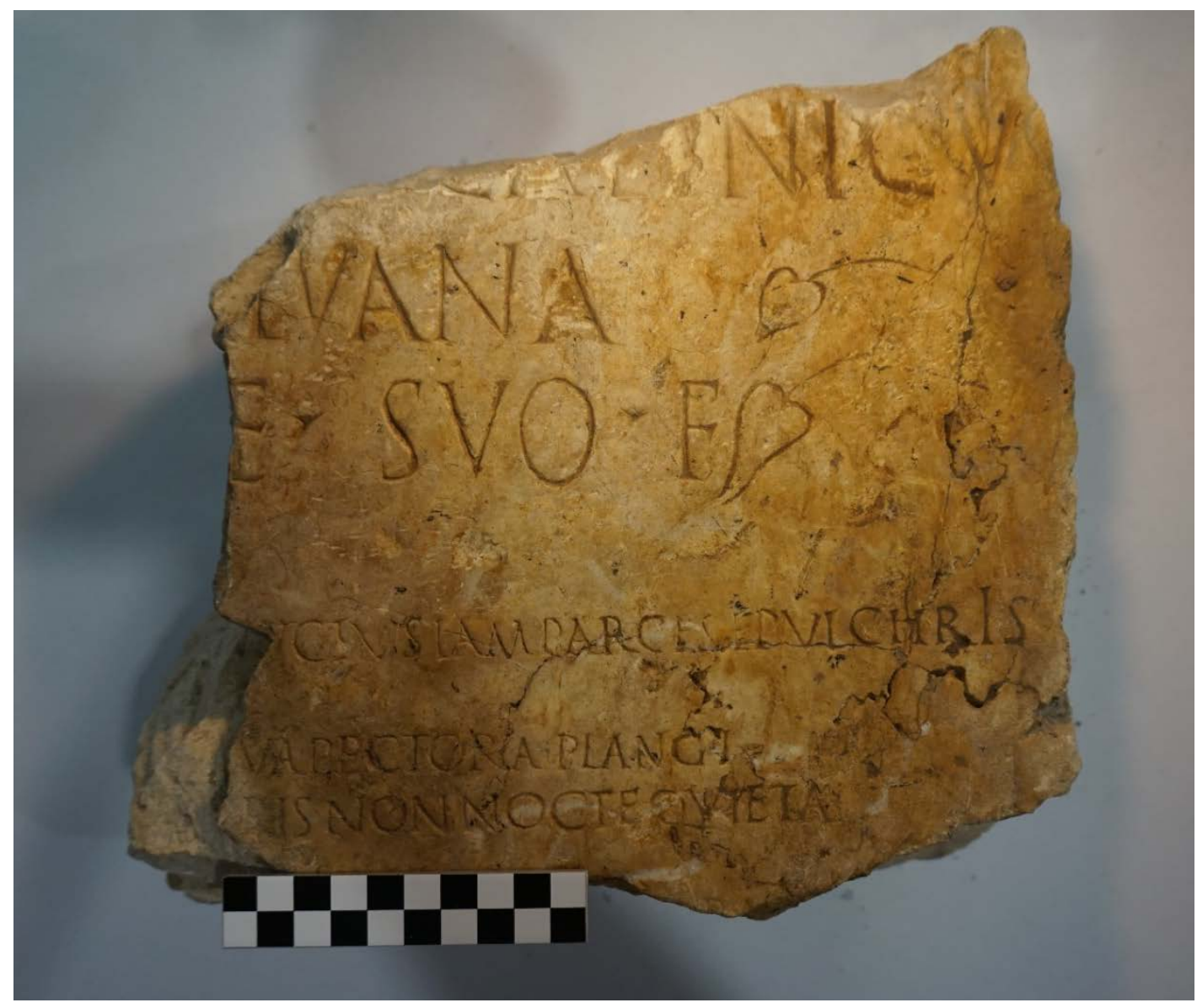

Figura 1: Inscripción completa. 


\section{EL ENCABEZAMIENTO}

La primera parte del texto aloja el encabezamiento con la dedicatoria funeraria de dos personajes enunciados en nominativo, Callinicus y Silvana, que erigieron el monumento de suo. Esta fórmula, habitual en epigrafía funeraria, aunque no exclusiva, aparece en diversos epígrafes de Tarraco acompañada del mismo verbo (fecit o fecerunt), ya desde época republicana $\left(C I L \mathrm{II}^{2} / 14,1314\right.$, de finales del s. II a.C. o inicios del I a.C.). ${ }^{3}$ La rotura de la pieza impide saber si en la parte perdida se hallaba el nombre de un tercer personaje, en dativo, al que podría haber estado dedicado el monumento, tal como aparece en los casos tarraconenses aludidos.

Tampoco es posible saber los gentilicios de los personajes, ya que solo se han conservado los respectivos cognomina -o nomina singularia, en el caso de que se tratara de esclavos. Sin embargo, la calidad de la inscripción nos inclina a pensar de que se trata de libertos o ingenui. En cuanto a los dos nombres recordados, Callinicus se documenta aquí por vez primera en Tarraco, aunque también es muy raro en el resto de Hispania, donde solamente aparece registrado en Valentia. ${ }^{4}$ Mejor representada está la forma femenina Silvana, mucho más común, también presente en Tarraco, que cuenta con tres testimonios referidos a una misma mujer (Valeria Silvana, CIL II²/14, 1232-1234, de inicios o mediados del II d.C.). ${ }^{5}$

\section{EL POEMA FUNERARIO}

La segunda parte del texto contiene un poema funerario, cuidadosamente inciso por una mano diversa, como demuestran el diferente ductus y el estilo paleográfico, probablemente obra de un lapicida especializado en este tipo de inscripciones (fig.2). ${ }^{6}$ La distribución de las tres líneas mantiene una ordinatio verso a verso, pero a causa de la rotura de la piedra solo se lee completa la segunda mitad de tres hexámetros dactílicos perfectamente compuestos. Sin embargo, se puede apreciar la calidad de la técnica poética, ya que el texto conservado incluye las características cesuras métricas, en este caso la heptahemímera, localizada en el primer y tercer verso:

\footnotetext{
${ }^{3}$ Otras inscripciones con la fórmula de suo fecit/fecerunt, ya de época imperial: CIL II $2 / 14,1043$; $1247 ; 1273 ; 1452 ; 1572$. Todas son de tipo funerario.

${ }^{4}$ Se trata de una dedicatoria a Serapis por la salud de su patrono P. Herennius Severus (CIL II $2 / 14$, 9). El nombre Callinicus, de origen griego, está bien documentado en el imperio romano occidental, aunque no abundantemente (65 entradas en una búsqueda general con la base de datos EDCS).

${ }^{5}$ La versión masculina está un poco mejor documentada en la ciudad, con cinco inscripciones (CIL II $2 / 14,1147-1148-1178 ; 1212 ; 1350$, referidas a tres personajes distintos), sin contar los sellos sobre ánforas Dressel 2-4 con la marca Silvan(i) recuperadas en el territorio (IRAT 156 a-c).

${ }^{6}$ Tarraco presenta en su rico corpus epigráfico diversas inscripciones en verso, de las cuales algunas incisas con especial cuidado. Por ejemplo, CIL II $2 / 14,1810=$ CLEHisp 160 (I d.C.); CIL $\mathrm{II}^{2} / 14,1279$ = CLEHisp 117 (fin. II d.C.); CIL II $2 / 14,1281$ = CLEHisp 117 (II d.C.) y CIL II $2 / 14,1287$ = CLE 1279 (fin. II-ini. III d.C.). A ellas cabe añadir las inscripciones indicadas en la nota 2 (ambas del s. I d.C.).
} 
[--- l]ūctūs || iām pārcĕ sĕpūlchrīs

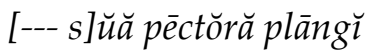

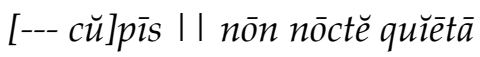

[--- f]ōrtū [na? ---]++[---]

Aunque breve, la secuencia poética permite identificar una serie de elementos comunes con otras composiciones epigráficas en verso que se alimentan directamente de los auctores latinos del repertorio hexamétrico clásico, principalmente Virgilio y Ovidio, pero no solo, como se verá a continuación.

\section{1. [--- l]uctus iam parce sepulchris}

Parece bastante claro reconocer la primera palabra como luctus, tanto por la coherencia temática con un contexto funerario como por su asiduidad en este tipo de carmina epigráficos. Su uso como plural poético aparece atestiguado en varias inscripciones, ya sea en plural: deponam luctus (CLE 1208); renovas magnos ...luctus (CLE 1404); duplices luctus (CLE 1210); mater quos natae debebat tradere luctus (CLE 2125), como en genitivo: et nostri memorem luctus (CLE 270); solamen tanti... luctus (CLE 1347); parva tibi... solacia magni luctus (CLE 2099); restant solamina luctus (CLE 1401); ponimus hunc / titulum luctus solacia nostri (CLEHisp 115); nunc eris... luctus causa mei (CLE 1107), y también en forma de sintagma preposicional: per luctus reddidit ipsa animam (CLE 1076).

Por su parte, la secuencia del adverbio iam seguido de imperativo también es un recurso documentado (iam liqui parentes, CLE 421), e incluso asociado al verbo parco (parcite iam lachrimis, CLE 1206) y a luctus: manes / parcite iam luctu sollicitare meos (CLE 1198). Es decir, la asociación de iam con el imperativo de parco y luctus forma parte del repertorio compositivo de tono funerario. Sin embargo, el final del verso alude, en realidad, el tópico del parce tumulo, es decir, la súplica para la protección del sepulcro para garantizar su inviolabilidad (Hernández Pérez 2001: 256-260). La forma con tumulo/tumulis está bien documentada en Roma e Italia (por ejemplo, parce tumulum Narcissi: CIL VI, $9274=$ CLE 1883), pero poco en Hispania, con un solo testimonio en Corduba (parcatis tumulo: CLEBetica CO9 = CLEHisp $26=$ HEp 2007, $\left.171=C I L I^{2} / 7,569\right)$, mientras que la variante con sepulchrum, documentada en plural en Tarraco, ${ }^{7}$ solo aparece en singular en una inscripción procedente de Hispellum (da quicumque legis fletus et parce sepulcro: CIL XI, 5335 = CLE 1813, hexámetro) y, reconstruida, en otra de Roma (patrias admitte preces et parce s[epulcro]: CLE 857).

A parte del caso de Tarraco, en Hispania solo se conocía la fórmula parce sepult[---] en un fragmento de inscripción hallado en la antigua Celsa (Velilla de Ebro) (HEp. 10, 2000, $638=$ CLEHisp 141) (fig.3). La secuencia se inspira directamente en el verso virgiliano quid miserum, Aenea, laceras? iam parce sepulto (Aen. 3.41. Cf. Hoogma 1959: 248) y, de momento es el único testimonio que

\footnotetext{
${ }^{7}$ Su empleo en plural puede ser entendido como licencia poética: "apiádate de las tumbas", es decir, "respeta esta tumba". Sobre el tópos del ne sepulcrum violetur y otras fórmulas de imprecatio para mantener la integridad de las tumbas, véase Hernández Pérez 2001: 256-260.
} 
transcribe el paso de Virgilio de manera literal. ${ }^{8}$ Además, está datado por contexto arqueológico en época augústea, ${ }^{9}$ lo que convierte a la inscripción de Celsa en uno de los documentos más antiguos que prueban la transmisión epigráfica de la obra del autor. ${ }^{10}$

\section{2. [--- s]ua pectora plangi}

El segundo fragmento de verso conserva una secuencia atestiguada prácticamente sin variaciones en un par de inscripciones de Roma: maeret et ad cineres plangit sua pectora palmis / nec satis ereptam lucem sibi (CLE 398) y quid mater ventrem laceras? Quid pectora plangis? / cavere fatum nemo (CLE 2155). ${ }^{11}$

La cláusula formada por pectora (plural poético de pectus) y el verbo plango es de clara matriz ovidiana. Aparece cinco veces en Heroidas: rupique sinus et pectora planxi (5.71); tunc demum pectora plangi contigit inque meas unguibus ire genas $(11,91)$; abscissa planxi mea pectora veste (12.153); consuetaque pectora plangit (13.491) y postquam se dolor invenit, nec pectora plangi nec puduit scissis exululare comis (15.113); dos en las Metamorfosis: laniataque pectora plangens (6.248); consuetaque pectora plangit (13.491); dos más en los Fasti, aunque se trata de la misma estructura repetida: pectore plangit humum (Fast. 1.578; 4.896) y una en Amores: plangite pectora pinnis (2.6.3). También se constata su uso en otros autores, aunque en menor medida, ${ }^{12}$ por lo que no hay duda de que tanto el verso del carmen de Tarraco como los de los paralelos romanos beben directamente de Ovidio. ${ }^{13}$

\section{3. [--- cu]pis non nocte quieta}

La erosión sobre la superficie deja abierta la posibilidad de leer culpis en lugar de cupis. Sin embargo, la forma culpis no aparece documentada epigráficamente, aunque sí, en cambio, en singular (Concordanze: 127), mientras que el verbo cupio enunciado en su segunda persona es habitual y coherente con el estilo dialogante

8 [---?] quicumque es [---] / [---? pa]rce sepult[---]. La inscripción fue publicada por M. Beltrán Lloris (1998: 128-129, n. 347); no obstante, la lectura [---pa]rce sepult[---] se debe a la revisión de F. Beltrán Lloris (2002: $624=H E p .10,2000,638)$, quien, sin embargo, no advirtió la coincidencia con Virgilio ni los paralelos con los CLE de Roma e Hispellum, que sí puso en evidencia P. Cugusi en su revisión de los CLE hispanos (2012: 106 y 191, n. 141).

9 “Hallado en una calle, en contexto datable entre el 27 a. E. y 14 d. E.” (Beltrán Lloris 2002: 624).

${ }^{10}$ Junto con el importante testimonio que representa la localización de un verso de la Eneida (2.268) en la pared de la cueva de Peñalba de Villastar (Teruel) (CLEHisp 139 = CLE Hispaniae TE1), antiguo santuario celtibérico, lo que confirma la efectiva implementación del modelo educacional romano entre la población indígena de la Citerior, incluso en zonas rurales del interior, en época imperial temprana (Díaz Ariño et al. 2019: 411; Díaz Ariño, e.p.).

${ }^{11}$ Otro poema reza: praesulis in viscibus clausisti pectora saeva, I ne mandata patrum perderet (CLE 1382).

${ }_{12}$ Por ejemplo, en Propercio: plangas pectora nuda (2.24b.52), Marcial: Plange, Favor, saeva pectora nuda manu (10.50. 2) y Estacio: iugentes iterasti pectore planctus (Silv. 3.5.53).

${ }^{13}$ Para Reeson (2001: 92) se trata de una yuxtaposición que, sin duda, se debe más a una frase con intenciones onomatopéyicas que a razones métricas. 
de este tipo de textos (Pepe, e.p.). Por ejemplo, hoc qui scire cupis iaceant quae membra sepulchro (CLE 1449).

En cuanto a la secuencia nocte quieta, es una variante participial de la forma personal documentada en una inscripción de Roma: placida... / nocte quiescant (CLE 467) y en un grafito pompeyano: felicem somnum qui tecum nocte quiescet (CLENuovo: 35). Sin embargo, nocte quieta parece remitir literalmente a un verso de las Argonáuticas de Valerio Flaco: gaudet Averna palus, gaudet iam nocte quieta (6.158). Este autor no es muy habitual entre los auctores de CLE, ya que apenas se ha propuesto vislumbrar una alusión a Arg. 7.244 (nulla quies animo, nullus sopor) en un grafito del Palatino (CLE 947. Cf. Cugusi 1996: 359; Cugusi 2007: 176). En consecuencia, el nuevo epígrafe de Tarraco podría ser considerada la primera cita literal de este autor en soporte epigráfico conocida hasta el momento.

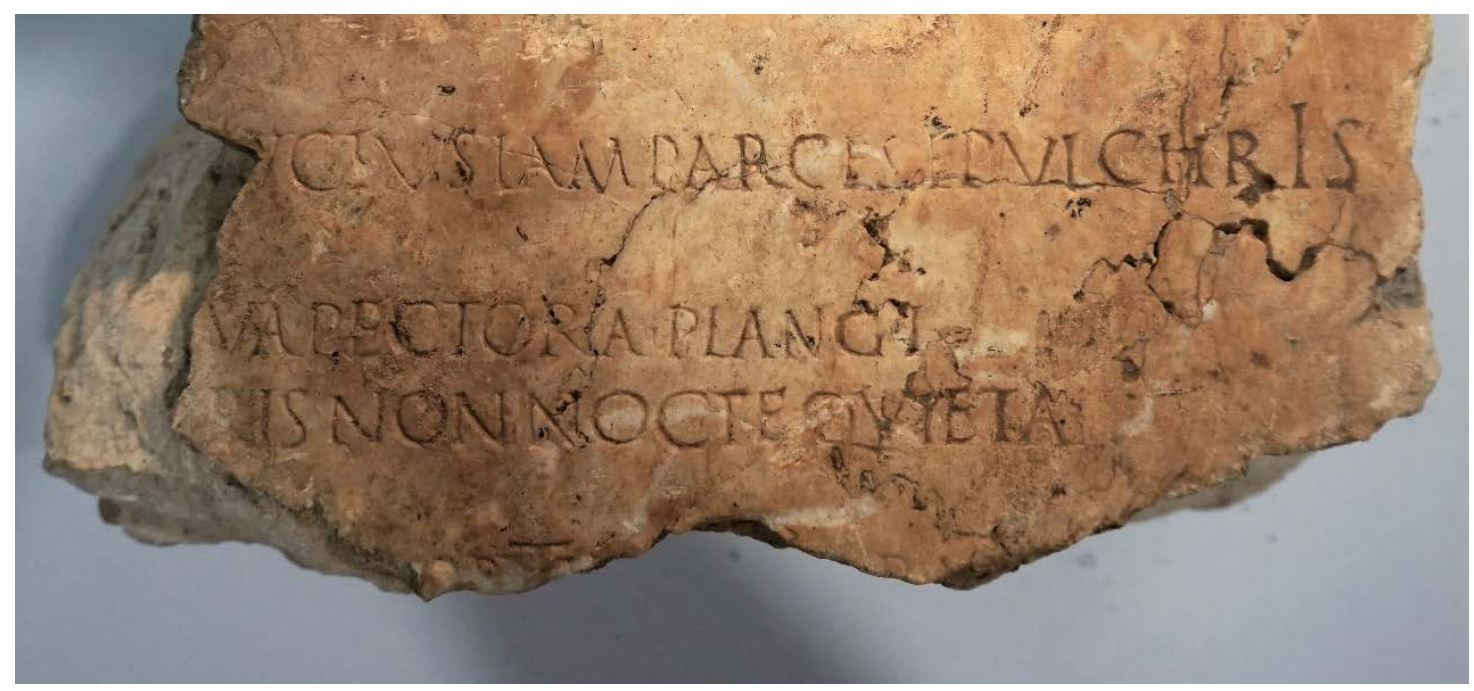

Figura 2: Detalle de la segunda parte del texto. Se pueden apreciar los diversos espacios de separación entre el encabezamiento y la parte con el carmen epigraphicum, así como entre los diversos versos del poema. Observese también los restos de letras de la cuarta línea, entre los que destaca la T montante.

Finalmente, la aliteración formada por el adverbio non seguido de palabra iniciada por no- se documenta en una inscripción de Roma (non nosse silentium: CLE 1109; cf. non nunc: CLE 970-971) y en otra de Arles (non nosti amicos: CLE 470). ${ }^{14}$ La secuencia non nocte aparece en un verso de Silio Itálico (non nocte dieve: Pun. 8.648), pero también en autores como Cicerón y, especialmente, Livio que la emplea reiteradamente como recurso estilístico (non nocte, non die). ${ }^{15}$

4. [---f]ōrtū[na?----]++[---]

${ }^{14}$ Así aparece también como recurso en el poema tardío de Porta Latina que inicia non nomen non quo genitus non unde quid egi (Massaro 2018: 133).

${ }^{15}$ Véase, por ejemplo, las varias citas en Livio para las expresiones non nocte, non die (21.11.5.3; 32.15.2.1), non nocte remissus (6.4.10.2); frente a la única mención en Cicerón (non parietes conscios, non nocte illam funestam ac luctuosam perhorrescet?, Cael. 60.7). 
El último verso se encuentra completamente afectado por la rotura de la piedra, por lo que la única secuencia conservada es de difícil comprensión. Entre las diversas opciones para la integración de la laguna podría preferirse la palabra fortuna por su amplia presencia en los CLE (cf. Concordanze: 278-279), seguida, aunque a distancia, por mortuus y derivados (Concordanze: 488).

Una vez analizado el contenido de la parte conservada de los versos, es posible proponer una traducción aproximada:

[--- l]ūctūs || iām pārcĕ sĕpūlchrīs

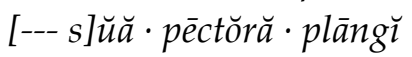

[--- cu]pīs I | nōn nōctě quǐètā

[--- f]ōrtū[na? ---]++[---]

“... del luto (?), respeta ahora los sepulcros / ... golpear su pecho / ...

deseas, no en la noche tranquila /... la fortuna (?)..."

Como conclusión, es posible poner de manifiesto la importancia de la nueva inscripción de Tarraco, cuya paleografía y contenido, así como el material empleado, permiten fechar la inscripción a finales del s. I d.C. Los fragmentos poéticos conservados transcriben de una manera muy cercana, dos de ellos literal, cláusulas de versos de la tradición hexamétrica de los clásicos Virgilio y Ovidio, a los que se puede añadir ahora Valerio Flaco, lo que junto a su correlativa presencia en los tres versos conservados permite plantear con bastantes visos de probabilidad que el carmen epigraphicum de Tarraco fue compuesto según la técnica del centón literario (cf. Polara 1990). Los dos primeros están sobradamente documentados como auctores de máxima referencia para los compositores de CLE hasta época Flavia (cf. Gómez Pallarès 1995), mientras que Valerio Flaco es un autor apenas documentado epigráficamente. Por otro lado, cabe destacar la variante tarraconense parce sepulchris del topos virgiliano, que se añade ahora a los más tempranos testimonios epigráficos de la recepción de este autor en Hispania (Celsa y Peñalba de Villastar). Por último, el nuevo carmen permite reconocer la influencia de Valerio Flaco entre los referentes de los compositores de poesía funeraria en piedra. Además, la cronología propuesta para la inscripción coincide con la fortuna de este último autor en Roma, lo que contribuye a poner de manifiesto la temprana difusión de las Argonáuticas fuera de la península itálica. 


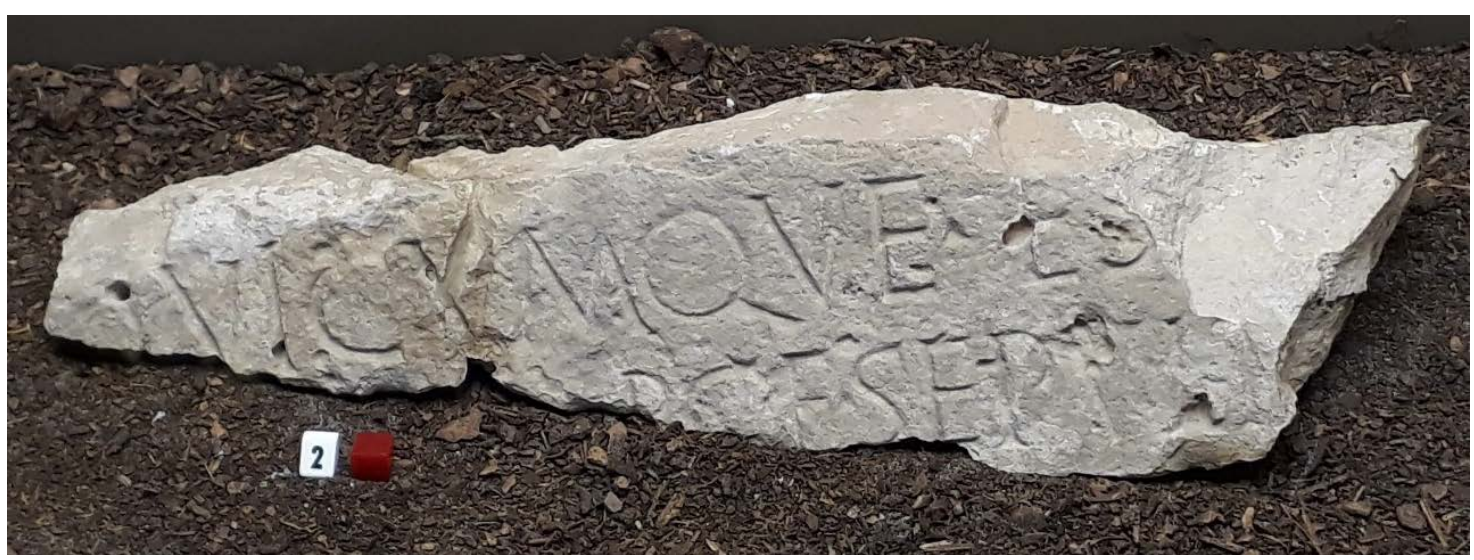

Figura 3: Inscripción de Celsa (Velilla de Ebro) (HEp. 10, 2000, 638 = CLEHisp 141). Museo de Zaragoza. Sección de la Colonia Celsa (Velilla de Ebro).

(Foto de la autora por cortesía del Museo).

\section{BIBLIOGRAFÍA}

BELTRÁn LlORIS, F. (2002), “Epigrafía latina en Aragón (II) (con un apéndice sobre la epigrafía paleohispánica)", Caesaraugusta, 75, 593-656.

BELTRÁN LlORIS, M. (1998), “Museo de Zaragoza. Colonia Celsa. Catálogo Monográfico", Boletín de Museo de Zaragoza, 14, 5-172.

CiURANA, J. (2011), “Prácticas y rituales en las áreas funerarias del suburbio oriental de Tarraco", en ANDREU, J., ESPINOSA, D. y PASTOR, S. (eds.) Mors Omnibus instat. Aspectos arqueológicos, epigráficos y rituales de la muerte en el occidente romano, , Madrid, Liceus, 331-350.

CLE = BÜCHELER, F. y LOMMATZSCH, E. (1930), Carmina Latina Epigraphica, Leipzig, Teubner.

CLEBetica = FERNÁNDEZ MARTÍNEZ, C. (2007), Carmina Latina Epigraphica de la Bética romana. Las primeras piedras de nuestra poesía, Sevilla, Universidad de Sevilla.

CLE Hispaniae = FERNÁNDEZ MARTínez, C., GÓMES PAllarès y DEL HoYo, J. (eds.), Carmina Latina Epigraphica Hispaniae. Portal de poesía epigráfica latina: búsquedas icónicas y textuales (http://cle.us.es/clehispaniae/index.jsf).

CLEHisp = CUGUSI, P. (2012), Carmina Latina Epigraphica Hispanica post Buechelerianam collectionem editam reperta cognita, Faenza, Fratelli Lega.

CLENuovo = Cugusi, P. (2007), Per un nuovo Corpus dei Carmina Latina Epigraphica. Materiali e discussioni, Roma, Atti della Accademia nazionale dei Lincei, 2007.

CONCORDANZE = COLAFRANCESCO, P. y RICCI, M. L. (1986), Concordanze dei Carmina Latina epigraphica, Bari, Edipuglia.

Cugusi, P. (2002), “Tradizione elegiaca latina e Carmina latina. Epigraphica. Letteratura e testi epigrafici", Aufidus, 48, 17-29.

Cugusi, P. (2007), Per un nuovo Corpus dei Carmina Latina Epigraphica. Materiali e discussioni, Roma, Atti della Accademia nazionale dei Lincei, 2007.

Cugusi, P. (2013), “CLE 1988 (= CIL VI, 37965)", l'epigramma longum e l'elegia. Qualche osservazione metodologica sui testi epigrafici versificati", Epigraphica, 75, 233-249. 
DÍAZ ARIÑO, B., ESTARÁN, M. J. y SIMÓN, I. (2019), “Writing, colonization, and Latinization in the Iberian peninsula", en SINNER, A. G.; VelAZA, J. (eds.), Palaeohispanic Languages and Epigraphies, Oxford, Oxford University Press, 396-416.

DÍAZ ARIÑO, B. (e.p.) “Culture écrite, latinisation et épigraphie dans l'Hispanie Citérieure républicaine" en Coltelloni-Trannoy, M. y MONCuniLl, N. (eds.), La culture de l'écrit en Méditerrannée occidentale à travers les pratiques épigraphiques, Lovaina, Peteers.

EDCS = Epigraphik-Datenbank Clauss / Slaby (http://www.manfredclauss.de/)

GÓMEZ PALLARÈS, J. (1995), "Cultura literaria en el corpus de los CLE Hispaniae hasta época flavia", en BELTRÁN LlORIS, F. (ed.), Coloquio sobre Roma y el nacimiento de la cultura epigráfica en occidente. Zaragoza, 4 a 6 de noviembre de 1992, Zaragoza, 151-162.

HERNÁNDEZ PÉREZ, R. (2001), Poesía latina sepulcral de la Hispania romana: estudios de los tópicos y sus formulaciones, Valencia, Universidad de Valencia.

Hoogma, R. P. (1959), Der Einfluss Vergils auf die Carmina latina epigraphica, Amsterdam, North-Holland publishing Company.

IRAT = D. GOROSTIDI (2010), Ager Tarraconensis 3. Les inscripcions romanes (Documenta 16), Tarragona, Institut Català d'Arqueologia Clàssica.

LIMÓN, M. (2014), La compaginación de las inscripciones latinas en verso. Roma e Hispania, Roma, L'Erma di Bretschneider.

MASSARO, M. (2013), "L'impaginazione delle iscrizioni latine metriche o affettive", Atti della Pontificia Accademia Romana di Archeologia, Rendiconti, 85, 2012-2013, 365-413.

MASSARO, M. (2018), "Questioni di autenticità di iscrizioni metriche (o affettive)", in: GALLO F. y SARTORI A. (eds.), Spurii lapides. I falsi nell'epigrafia latina, Milán, Biblioteca Ambrosiana, 93-135.

PEPE, C. (e.p.), "Voci in dialogo nella poesia sepolcrale latina: strumenti testuali ed extratestuali", en FERNÁNDEZ MARTíNEZ, C. y LIMÓN BELÉN, M. (eds.), V Reunión Internacional sobre Poesía Epigráfica Latina, Sevilla, Universidad de Sevilla, e.p. [versión provisional online]

Polara, G. "I Centoni”, en Cavallo, G., Fedeli, P. y Giardina, A. (eds.) Lo Spazio Letterario di Roma Antica, vol. 3: La Ricezione del Testo, Roma, Salerno Editrice, 245-275. REESON, J. (2001), Ovid Heroides 11, 13 and 14: a commentary, Leiden-Boston-Köln, Brill. 